have never seen one case in danger; while the secondary cases of small-pox which I have seen have been far more severe, and two have died; thus establishing my position of the greater security afforded to the patient from the malignant consequences of smallpox. To this I should add, that during the last thirty years I have had nine pupils and six assistants; these had all been vaccinated; when a case of small-pox has occurred, they have been invariably anxious to watch over such case most sedulously, for the sake of acquiring a knowledge of what is now a rare malady; and, desirous of testing their own security; they have sat for hours in the patient's confined room at the pest-house; they have breathed the patient's breath, and laid down upon lis bed for a longer or shorter time; and yet what has been the result? Perfect immunity! The family at the pest-house also had been vaccinated, yet no instance of failure occurred. These, however, were young; not so my pupils and assistants, but ranging from sixteen to thirty. Let it not be said that they possessed the freedom from susceptibility, which is given by professional fearlessness, for scarcely one, if one, escaped scarlatina during their residence with me. Fact against fact. I am indeed aware that the number of cases is too small to admit of general inference, but I do not hesitate to affirm, that such a result could not have happened to the unprotected. "Valeat quantum valere possit."

I will not occupy your space by copying Mr. Brown's fourth conclusion, and only very briefly notice its extraordinary contents. He first states, that cases of small-pox after vaccination were not heard of for the first few years after its introduction; and hence wishes to infer, that the influence of vaccinia is temporary, as if such inference were at all justifiable; and as if, in fact, the phenomenon, if true, were not much more readily accounted for by the paucity of cases which, at that early period, could be subjected to variolous infection.

I admit that, from the multiplication of vaccinated persons, especially of individuals carelessly and imperfectly vaccinated, and from the presence of variolous epidemics, the number of cases of failure has apparently increased; but I take leave to deny his assertion, and challenge him to the proof, "that the fatal effect of small-pox succeeding to vaccination actually exceed (exceeds) the proportion of twenty to one those which were found to attend the practice of inoculation." I suppose it is intended to say, that death occurs with greater frequency in the former over the latter cases in the proportion of twenty to one. Now, as the proportion of deaths from epidemic small-pox has usually been, in round numbers, about twenty-five in one hundred-and that from inoculated small-pox, about four in one hundred-it will follow that this number multiplied by twenty, will give the proportion of deaths from small-pox after vaccination as eighty in one hundred !!! We challenge Mr. Brown to the proof. We are quite ready to allow the ravages of small-pox upon the unprotected, and to bewail the indifference which has been produced amongst the poor towards the practice of vaccination, partly and chiefly from their belief of the great infrequency of small-pox, and partly from their prejudices, fostered and preserved by the designing and interested inocu- lator, or by the perverse and misguided sons of intellect and science, such as Mr. Brown.

On the subject of Mr. Brown's fifth conclusion, I am only prepared to say, that I believe similar errors and imperfections attend the theory and practice of vaccination in other countries as well as in our own; the reasoning, therefore, which is applicable to the one is applicable to the other.

With regard to the last conclusion, we readily yield to Mr. Brown, that it is absurd to contend for the absolute security afforded by vaccination; none but the most unwary and injudicious friends of vaccination contend for its giving perfect immunity. We also yield the point as to the comparative frequency of small-pox after small-pox, and small-pox after vaccination, although it is not easy to institute a comparison between the few inoculated, and the many vaccinated, of the present generation. But we can afford to yicld the point-what we contend for is, simply, that the mortality in the cases of small-pox after small-pox is greater, cœteris paribus, than in small. pox after vaccination.

I trust you will forgive me for having occupied so much of your space in combatting Mr. Brown's conclusions. On a subject of such vital importance to the community as well as to ourselves it appears to me desirable to show the illogical reasonings of my antagonist, and, if you are not tired with your correspondent, I shall hope to resume the subject next week.

I am, yours faithfully, W. Newnhas.

May 10, 1842.

\section{ON THE RADICAL CURE}

OF

\section{REDUCIB LE INGU INAL HERNIA.}

\author{
By Dr. Carl Haller, Vienna.
}

CASE I.-Joseph S., aged 23, was affected with inguinal hernia on the right side; the tumour was about the size of a pigeon's egg, and easily reducible. Such was the state of the patient in the year 1837. In May, 1840, he was admitted into hospital, for the purpose of undergoing an operation. The tumour was now as large as a hen's egg, and contained a small coil of intestine; the external abdominal ring was sufficiently enlarged to admit the end of the index finger.

On the 26th of August, 1840, the bowels having bcen previously emptied by an enema, the operation was performed in the following manner:-The skin of the scrotum was pushed up, with the index finger, into the inguinal canal, and, with a sonde a dard, two ligatures were passed through it and the walls of the canal. A compress of lint was now passed up as high as possible into the canal, and the ligatures firmly tied over it on two quills covered with sticking plaster. The part was covered with ice.cold lotions, the scrutum supported with a suitable bandage, and the patient ordered to keep perfectly quiet in bed. The pain which followed was not very severe, but the parts became much swollen and hard, and on the seventh day there was some slight fever. The liga- 
tures were now divided and the compress removed. The discharge of pus ceased on the fourteenth day. On the twenty-eighth day an clastic truss was applied, and the patient allowed to move about a little. The to abdominal ring was, at this period, very small, and no protrusion of the tumour occurred when the patient coughed violently. Ten days afterwards the patient left the hospital and returned to his usual employments. The use of the truss was continued for several months, and on the tenth month after the operation the patient was dismissed from the house of correction perfectly cured.

CaSE II.-Joseph S., aged 19, was admitted into hospital on the 26th of October, 1810. He had laboured under a left inguinal hernia for five years; for twelve months he wore a truss, but then left it off. The tumour only descends in the upright position, is the size of a hen's egg, and is easily reduced. The operation was performed on the 28th of October in the manner already described.

On the 3rd of November, the tumefaction and red. ness extended up for several inches above the ligatures, and some pus escaped from the latter. The ligatures and plug were now removed and tepid water injected into the cavity, which discharged a good deal of pus.

Nov. 14. The suppuration has ceased, and the ligature wounds are healed, but the invaginated integument is beginning to sink down. On the 24th the integument had completely descended beyond the external ring, which, however, was much smaller than before the operation; the hernia did not come down on coughing or pressure being made. Three months after this the patient was attacked with severe catarrh, and coughed violently, but had no relapse of his rupture. In this favourable state he continued for six months, and thought himself cured, when, on the seventh month after violent straining at stool, the hernial tumour descended as before.

In the third case, also, the cure was only temporary, the gut having escaped on the fourth month after the operation.

Cass IV.-Gustavus T., aged 26, had suffered from inguinal hernia of the left side during four years. The tumour was the size of a hen's egg, easily reducible, and the ring admitted the end of the index finger.

The operation was performed, in the usual way, on the 15th of March. Very slight reaction took place; on the fourth day there was a little fever; the ligatures, \&c., were removed on the seventh day; the discharge of pus ceased on the fourteenth day; on the twentyeighth day the patient was allowed to leave his bed, being furnished with a truss; the external ring was not obliterated at this time, although, on passing the finger into the canal, a slight impediment could be felt ; the tumour did not appear on violent coughing or exertion. The patient left the hospital on the 19th of April, and was discharged from the house of correction on the 30th of August (six months after the operation), without having suffered any relapse.

CASE V.-Michael C., aged 27, had a hernial tumour on the right side, as long back as his recollec. tion goes. He was admitted on the 17 th of February, 1811 ; the tumour was as large as a goose's egg ; composed evidently of intestine, and easily reduced. The operation was performed on the 19th of February; soon afterwards the epigastric region became painful, but this subsided quickly, and the pain was confined to the part operated on. On the fourth day some slight febrile action took place. The ligatures and quills were removed on the sixth day, and the plug on the following day. On the twenty-eighth day the patient went about with a truss. The invaginated portion of integument had now completly come down, but the external abdominal ring was considerably narrowed, and the hernial tumour did not appear on coughing or exertion. The patient was dismissed from hospital on the 5th of April, and was employed in various laborious occupations until the 25th of August, 1811, when he was discharged from prison, without having experienced the slightest return of his hernia.

CASF $_{\text {A }}$ V1.--Johann F., aged 38, a strong, healthy man, had congenital inguinal hernia of the left side. When admitted into hospital on the 30th of July, 1841, the tumour was as large as a man's fist, elastic, and easily reduced. The operation was performed on the 6th of August without any difficulty.

In this case the reaction was greater than in any of the former. There was considerable tumefaction and pain, sense of oppression at the stomach, and some fever during the two days which immediately followed the operation. Castor oil was administered, and great relief followed the evacuation of the bowels. The ligatures and quills were removed on the sixth day; after the third week the discharge of pus has completely ceased. The results of the operation were, however, only temporary; for about eight weeks afterwards the intestine came down again, and the hernial tumour soon acquired its former volume.

CASE VII.-Franz R., aged 35, was affected with inguinal hernia of the left side, from the age of seven years. The tumour in this case was large, and the external ring very much dilated. The patient underwent the operation on July 19th, and on the twentycighth day was allowed to go about with a truss. The ring was now very much contracted, and the tumour did not come down when the patient coughed or made strong exertion. He was discharged six months afterwards without having suffered any relapse of his complaint.

During the past year, Dr. Günther has performed the operation of hernioraphy twelve times, on young and healthy' individuals, affected with moderate sized hernia; the method of operating selected was that recommended by Schuh. All the patients left the hospital cured, and, in several, the cure was ascertained to have been permanent after a lapse of several months. There is no doubt, however, that an essential condition of cure is the remaining quietly in bed for four wceks at least, and to this few patients would submit, unless after an operation. - Medis. Jahrbücher, 1842.

The next annual meeting of the Association of Medical Officers of Hospitals for the Insane will be held at Lancaster on Thursday, the 2nd of June next, at twelve o'clock. 